\title{
Erişkinlerde büyüme hormonu tedavisi
}

\section{Growth hormone therapy in adults}

\section{Fatih Kılıçlı*, Hatice Sebile Dökmetaş}

Endokrinoloji Bilim Dalı (Yrd. Doç. Dr. F. Kılıçlı, Prof. Dr. H. S. Dökmetaş), Cumhuriyet Üniversitesi Tıp Fakültesi, TR-58140 Sivas

\section{Özet}

Büyüme hormonu sentezi yaşam boyu devam eden, anabolizan etkisi olan, lipoliz ve karbonhidrat metabolizmasını etkileyen bir hormondur. Bin dokuz yüz seksenlerin 2. yarısına kadar çoğu endokrinolog erişkinlerde görülen büyüme hormonu eksikliğinin klinik bir sorun olmadığını düşünmekteydi. Erişkinlerde görülen büyüme hormonu eksikliği yaşam beklentisinde azalma, kardiyovasküler morbidite ve prematür aterosklerozda artış, vücut kompozisyonunda değişiklik, osteoporoz, egzersiz toleransında azalma ve yaşam kalitesinde düşme ile ilişkilidir. Büyüme hormonu replasman tedavisi, erişkin tip büyüme hormonu eksikliği olan hipofizer yetmezliği olanlarda faydalı etkiler gösterir. Büyüme hormonu eksikliği olanlarda büyüme hormonu tedavisi ile yaşam kalitesi, kemik mineral dansitesi, vücut kompozisyonu, egzersiz toleransı, lipid profili ve kan şekeri gibi vasküler risk faktörlerinde olumlu değişiklikler olduğu gösterilmiştir. Çocukluk döneminde başlayan büyüme hormonu eksikliklerinde, erişkin boya ulaştıktan sonra, büyüme hormonu durumu geçiş döneminde yeniden değerlendirilmelidir. Böylece maksimum kemik ve kas artışı gibi tam somatik gelişimi sağlamak için $\mathrm{BH}$ replasmanına devam edilip edilmeyeceğine karar verilebilir.

Anahtar sözcükler: Büyüme hormonu eksikliği, tedavi

\begin{abstract}
Growth hormone secretion occurs throughout life and is known to have profound effects on anabolism, lipolysis, and carbohydrate metabolism. Until the second half of 1980's growth hormone deficiency in adult life was not considered as a clinical problem by most endocrinologists. Growth hormone deficiency in adults is associated with reduced life expectancy, increased cardiovascular morbidity and premature atherosclerosis, changes in body composition, osteoporosis, reduced exercise tolerance and reduced quality of life. Growth hormone replacement therapy has important beneficial effects in adult hypopituitary patients with growth hormone deficiency. It is shown that growth hormone therapy has a positive influence in growth hormone deficiency patients on quality of life, bone mineral density, body composition, exercise tolerance and vascular risk factors such as blood glucose levels and lipid profile. In patients with childhoodonset growth hormone deficiency, after cessation of linear growth, growth hormone status should be reevaluated in the transition age for continued growth hormone replacement to achieve full somatic development including the maintenance of maximal bone and muscle mass.
\end{abstract}

Keywords: Growth hormone deficiency, treatment

Geliş tarihi/Received: 18 Ekim 2012; Kabul tarihi/Accepted: 15 Kasım 2012

*İletişim adresi:

Dr. Fatih Kılıçlı, Endokrinoloji Bilim Dalı, Cumhuriyet Üniversitesi Tıp Fakültesi, TR-58140 Sivas. E-posta: mfatihkilicli@yahoo.com

\section{Giriş}

Yaklaşık 20 yıl öncesine kadar erişkin hipofiz yetmezliği olan hastalara sadece tiroksin, steroid ve gonadal hormon replasmanı yapılırdı ve pubertal büyüme tamamlandıktan sonra büyüme hormonu $(\mathrm{BH})$ eksikliğinin klinik bir sorun oluşturmadiğ düşünülmekteydi. Yapılan çok sayıda çalışma sonucu elde edilen veriler 1şığında erişkin tip $\mathrm{BH}$ eksikliğinin başta kardiyovasküler hastalık insidansında artış [1] olmak üzere kemik mineral yoğunluğunda azalma [2], vücut kompozisyonunda değişiklik [3], psikososyal sorun [4], yaşam kalitesinin düşmesi [5] gibi bir çok soruna neden olduğu gösterilmiştir. 


\section{Erişkinde BH eksikliğ i nedenleri}

1. Çocukluk döneminde başlayan BH eksikliği: Pediatrik dönemden erişkinliğe geçiş BH eksikliğinin yeniden dinamik testlerle değerlendirilmesi için uygun bir dönemdir. Multipl hipofiz hormon yetmezliği olan ve en az 1 aydır BH tedavisi almayan hastalarda IGF-I düzeyi düşükken, sellar veya suprasellar bölgede radyolojik olarak tespit edilen konjenital bir anomali, kraniyofarengioma gibi kazanılmış hipotalamo hipofizer yetmezlik, malign hastalık için hipotalamo hipofizer bölgeye yüksek doz radyoterapi uygulanması veya bu bölgedeki hastalıklar için cerrahi uygulanması, BH sentezini bozan genetik defekt gibi durumlardan biri varsa bu hastalarda kalıcı $\mathrm{BH}$ eksikliği riski oldukça yüksektir. $\mathrm{Bu}$ hastalarda kalıcı $\mathrm{BH}$ eksikliği olduğu kabul edilir ve erişkin dönem $\mathrm{BH}$ eksikliği tanısı koymak için provokasyon testi yapılması gerekmez [6]. Yukarıda bahsi geçen nedenler dişında $\mathrm{BH}$ eksikliği olan çocuklar idiyopatik $\mathrm{BH}$ eksikliği olan hasta grubunu oluşturmaktadır ve bu grup hastalar neredeyse tüm serilerde çocukluk döneminde başlayan $\mathrm{BH}$ eksikliğinin en sık nedenidir [7, 8]. Bu hastalar erişkin boyuna ulaştıktan sonra BH tedavisine devam etmeden önce yeniden dinamik testler ile BH eksikliği açısından değerlendirilmelidir çünkü idiyopatik BH eksikliği olan çocuklarda kalıcı BH eksikliği, yapısal nedenlere bağlı $\mathrm{BH}$ eksikliği olan hastalara göre daha düşüktür. İdiyopatik BH eksikliği olan çocuklar erişkin dönemde test edildiklerinde çoğu hastada BH cevabının yeterli olduğu görülmüştür [9]. Burada dikkat edilmesi gereken bir ayrıntı ise bu hastaların $\mathrm{BH}$ eksikliği için yeniden test edilmeden en az 1 ay önce $\mathrm{BH}$ tedavilerinin kesilmesidir [10]. Böylece $\mathrm{BH}$ tedavisi ile oluşacak supresyon önlenmiş olur.

2. Erişkin dönemde başlayan BH eksikliği: Hipotalamus ve hipofizin yapısal hastalı̆̆ olanlar (hipofiz adenomu, Ratke kisti, menengiom gibi), bu bölgelere cerrahi işlem veya radyoterapi alanlar, kafa travması öyküsü olanlar ve hipofiz yetmezliği tanısı alanlar erişkin tip $\mathrm{BH}$ eksikliği açısından değerlendirilmelidir. Erişkinlerdeki $\mathrm{BH}$ eksikliğinin en sik nedeni hipofiz adenomu veya bu nedenle yapilan operasyonlar veya radyoterapidir [6]. Makroadenomlar daha fazla oranda hipofizer yetmezliğe neden olurken (\%30-60) nonfonksiyone mikroadenomların da (\%42) BH eksikliğine neden olduğu gösterilmiştir [11]. Son dönemlerde yapılan çalışmalar sonucunda beyin hasarı (travmatik beyin hasarı, spora bağlı tekrarlaycı kafa travması ve subaraknoid kanaması) olan hastaların da hipofizer yetmezlik açısından değerlendirilmesi gerektiği ortaya çıkmıştır [12-14]. Özellikle şuur kaybı olan ve 24 saat hastanede kalan travmatik beyin hasarı olanlar travmadan 6-12 ay sonra hipofizer yetmezlik açısından değerlendirilmelidir [15]. BH'nun patolojik durumlarda ilk etkilenen hipofiz hormonu olduğu bilindiğinden bu hastalarda erişkin tip BH eksikliğinin ekarte edilmesi mantıklı bir yaklaşım olarak görülmektedir.

3. İdiyopatik BH eksikliği: Erişkinlerde nadir olduğu için bu grup hastalarda tanı koymak için en az 2 provokasyon testi ile tanı doğrulanmalıdır. Obezitesi olan erişkinlerde IGF-I düzeyindeki düşüklük tanının doğruluğunu destekleyen bir bulgudur [6].

\section{Tanı}

BH eksikliği tanısı için altın standart test ITT'dir. Çocuklarda pik $\mathrm{BH}>10 \mathrm{ng} / \mathrm{mL}$, erişkinlerde pik $\mathrm{BH}>5 \mathrm{ng} / \mathrm{mL}$ olması $\mathrm{BH}$ eksikliği olmadığını gösterir. ITT sonrası pik $\mathrm{BH}$ değeri $<3 \mathrm{ng} / \mathrm{mL}$ olması BH eksikliği tanısı koydurur [10] ITT kardiyovasküler hastalıklarda, epilepside ve serebrovasküler hastalığı olanlarda kontrendikedir. Endocrine Society'nin son klavuzunda GHRH-arginin testi'de ITT ile birlikte birinci seçenek olarak önerilmiştir ancak BH eksikliğinin sebebi radyoterapi gibi hipotalamik bir nedene bağlıysa GHRH-arginin testi yanıltıcı olabilir [16]. GHRH-arginin testi kiloya göre değerlendirildiğinde ITT kadar değerli bir testtir. Bu test sırasındaki pik BH düzeyi VKİ $<25 \mathrm{~kg} / \mathrm{m}^{2}$ olanlarda $11 \mu \mathrm{g} / \mathrm{L}$, VKI $25-30 \mathrm{~kg} / \mathrm{m}^{2}$ olanlarda $8 \mu \mathrm{g} / \mathrm{L}, \quad \mathrm{VKI}>30 \mathrm{~kg} / \mathrm{m} 2$ olanlarda $4 \mu \mathrm{g} / \mathrm{L}$ düzeyinden düşük ise $\mathrm{GH}$ eksikliği tanısını koydurur [6]. ITT'nin veya GHRH-arginin testinin uygun olmadığ 1 hastalarda $\mathrm{BH}$ eksikliği için glukagon stimulasyon testi kullanılabilir. Bu testte $\mathrm{GH}$ cevabi en az 3 saat süreyle izlenmelidir. Pik $\mathrm{BH}$ düzeyi $<2,5-3 \mu \mathrm{g} / \mathrm{L}$ ise $\mathrm{BH}$ eksikliği tanısını koydurur [6]. Provakatif testler ile cut-off 
değerlerin altında pik BH düzeyi olanlarda IGF-I düzeyindeki düşüklük BH eksikliği tanısını koydurur ancak kötü kontrollü diyabet, KC hastalığı, oral östrojen tedavisi ve anoreksi gibi durumlarda IGF-I düzeyinde azalma olabileceği akılda tutulmalıdır [6]. Obezlerde BH'na duyarlılık artmıştır. Bu nedenle obezlerde IGF-I düzeyindeki düşüklükler $\mathrm{GH}$ eksikliğini destekleyen bir bulgudur [10]. Hipofizer hormonlardan 3 veya daha fazla eksiklik olduğunda $\mathrm{BH}$ eksikliği olasılığı oldukça fazladır ve bu durumlarda IGF-I düzeyi de düşük ise provokasyon testi yapmaksızın tanı konulabilir [6].

\section{BH tedavisinin faydaları}

BH tedavisi ile vücut kompozisyonunda düzelme [17] ve egzersiz kapasitesinde artış [18] olduğu gösterilmiş̧ir. BH'u lipolizi artırarak total vücut yağını azaltır ve bu etki 6 aylık tedavi sonrası görülür. Tedavi devam ettiği sürece bu olumlu etki sürmektedir. BH eksikliğinde görülen yağsız vücut kitlesindeki azalma tedavi ile düzelir ve kas kitlesi artar [19]. Çocukluk döneminde başlayan $\mathrm{BH}$ eksikliğinde \%35, erişkin başlangiçlı $\mathrm{BH}$ eksikliğinde $\% 20$ oranında osteoporoz görülmektedir. $\mathrm{Bu}$ veriler $\mathrm{BH}$ eksikliğinin başlangıç yaşının osteoporoz gelişinde önemli olduğunu düşündürmektedir [6]. Bilinmesi gereken önemli bir ayrıntı da tedavinin 12. ayından önce yapılan kemik mineral dansitesinde (KMD) azalma görülebileceğidir [20]. Bu nedenle BH tedavisinin 18-24. ayında KMD ölçülmelidir [21]. BH eksikliği olan çocuklarda erişkin boya ulaşılsa bile iskelet ve kas maturasyonunun maksimum olması için geçiş dönemi boyunca BH tedavisine devam edilmesi önerilmektedir [6]. Yapılan çalışmlarda erişkin boya ulaştıktan 2 yıl sonrasına kadar BH tedavisi alan çocuklarda tedavi almayanlara göre KMD'de anlamlı artışlar olduğu gösterilmiştir [22]. Bu nedenle hastalar DEXA ile KMD açısından değerlendirilmeli eğer anormallik varsa en az 2 yıl daha $\mathrm{BH}$ tedavisine devam edilmelidir. $\mathrm{Bu}$ hastalarda eğer tedavi kesilmişse erişkin dozda $\mathrm{BH}$ tedavisi mümkün olan en kısa zamanda başlanmalıdır. BH tedavisi ile erişkin tip BH eksikliği olanlarda kardiyovasküler fonksiyonlarda düzelme olduğu gösterilmiştir. Bu olumlu etkiler endoteliyal fonksiyon [23], lipoprotein metabolizması [24], inflamatuvar kardiyovasküler belirteçler [24] ve myokard fonksiyonları üzerine BH'nun olumlu etkileri [25] sonucu oluşmaktadır. Alman KIMS kohort çalışmasında 10 yıllık hesaplanmış kardiyovasküler olay riski $\% 5$ bulunmuş ve 2 yıllık büyüme hormonu replasman tedavisi ile $\% 50$ oranında anlamlı azalma olduğu saptanmıştır [26]. Erişkin BH eksikliği olanlarda yaşam kalitesi (çocukluk döneminde başlayanlarda daha az belirgin) ve kognitif fonksiyonlarda bozulma bir çok çalışmada gösterilmiştir ve büyüme hormonu replasman tedavisi ile anlamlı düzelme olduğu saptanmıştır [27, 28]. Yaşam kalitesindeki düzelmeler özellikle ilk 3 ayda olmak üzere çoğu ilk 1 yılda görülür.

\section{BH tedavisi}

BH tedavisi çocuklarda BH eksikliği, böbrek nakli öncesi kronik böbrek yetmezliğine bağlı boy kısalığı, Turner sendromumdaki ve Prader-Willi sendromundaki boy kısalığı, idiyopatik boy kısalığı ve normal büyüme persantilini yakalayamayan gestasyonel yaşı küçük olan hastalarda endikedir [6]. Erişkinlerde ise BH eksikliğinde endikasyonu vardır ancak HIV enfeksiyonuna bağlı kaşeksi durumlarının da dahil olduğu pek çok yeni alanda onay almak için bilimsel çalışmalar devam etmektedir [29]. Erişkinlerde BH tedavisi bireyseldir. Tedaviye düşük dozlarla başlanır ve klinik cevap, IGF-I düzeyi ve yan etkiye göre doz ayarlaması yapılır. Başlangıç dozu 30-60 yaş arasındaki hastalar için 0,2-0,3 $\mathrm{mg} /$ gündür. Otuz yaşından küçük genç erişkinlerde ise $0,4-0,5 \mathrm{mg} /$ gün gibi daha yüksek dozlar önerilir. Pediatrik dönemden erişkin döneme geçen hastalarda ise daha yüksek dozlar ile tedaviye başlanması gerekebilir. Altmış yaşından büyüklerde ise 0,1-0,2 $\mathrm{mg}$ /gün gibi daha düşük dozlarla tedaviye başlanması önerilmektedir. Doz ayarlaması 1-2 aylık aralıklarla yapılır. IGF-I düzeyinin normal yaş ve seks uyumlu aralığın üst yarısında olması hedeflenir. Bu hastalarda hedeflenen klinik etki sağlanır ve yan etki görülmezse hasta 6 ayda bir IGF-I düzeyi ile takip edilir. BH tedavisinin ne kadar devam etmesi gerektiği hakkında oluşmuş bir konsensus yoktur. Eğer klinik fayda görülmüşse tedavinin kesilmesine gerek yoktur ancak 1 ylllık tedaviye rağmen fayda görülmemişse tedavinin 
kesilmesi önerilmektedir [6]. Oral östrojen tedavisi alanlarda hedef IGF-I değerlerine ulaşmak için daha yüksek dozlarda $\mathrm{GH}$ tedavisine ihtiyaç duyulur çünkü östrojen BH'nun postreseptör inhibisyonuna neden olarak IGF-I sentezini suprese eder [30]. Aynı nedenden dolayı kadınlarda erkeklere göre daha yüksek dozlara çıkmak gerekir. BH tedavisi alanlarda dikkat edilmesi gereken diğer ayrıntı tiroid ve adrenal fonksiyonların da değerlendirilmesidir. BH tedavisi serbest T4 düzeyinde ve kortizol düzeyinde azalmaya neden olur. Hipotiroidi ve adrenal yetmezlik nedeniyle tedavi alan hastalarda doz ayarlamas1 gerekebilir $[31,32] . \mathrm{BH}$ tedavisi öncesi hipotalamopituiter aks değerlendirildiğinde hormonal eksiklik olmasa bile $\mathrm{BH}$ tedavisi alırken steroid eksikliğinin gelişebileceği akılda tutulmalıdır [32].

\section{BH tedavisi ile ilişkili yan etkiler}

Yan etkiler hastaların \%5-18'inde görülür ve sıvı retansiyonu ile ilişkilidir. Periferik ödem, artralji, parestezi ve miyalji en sık görülen yan etkilerdir [33]. Retinopati [34], benign intrakraniyal HT [35] ve jinekomasti [36] ise nadir görülen komplikasyonlardır. Yan etkiler uygun doz titrasyonu ile düzelirler. BH tedavisi alan diyabetik hastalarda BH'nun insülin antagonisti etkisinden dolayı antidiyabet ilaçlarda da doz ayarlaması gerekebilir [6].

\section{Kaynaklar}

1. Rosén T, Bengtsson BA. Premature mortality due to cardiovascular disease in hypopituitarism. Lancet 1990; 336: 285-8.

2. Wüster C, Slenczka E, Ziegler R. Increased prevalence of osteoporosis and arteriosclerosis in conventionally substituted anterior pituitary insufficiency: need for additional growth hormone substitution? Klin Wochenschr 1991; 69: 769-73.

3. Beshyah SA, Freemantle C, Thomas E, Rutherford O, Page B, Murphy M, Johnston DG. Abnormal body composition and reduced bone mass in growth hormone deficient hypopituitary adults. Clin Endocrinol (Oxf) 1995; 42: 179-89.

4. Rosén T, Wirén L, Wilhelmsen L, Wiklund I, Bengtsson BA. Decreased psychological well-being in adult patients with growth hormone deficiency. Clin Endocrinol (Oxf) 1994; 40: 111-6.

5. Rosilio M, Blum WF, Edwards DJ, Shavrikova EP, Valle D, Lamberts SW, Erfurth EM, Webb SM, Ross RJ, Chihara K, Henrich G, Herschbach P, Attanasio AF. Long-term improvement of quality of life during growth hormone $(\mathrm{GH})$ replacement therapy in adults with GH deficiency, as measured by questions on life satisfaction-hypopituitarism (QLS-H). J Clin Endocrinol Metab 2004; 89: 1684-93.

6. Molitch ME, Clemmons DR, Malozowski S, Merriam GR, Vance ML; Endocrine Society. Evaluation and treatment of adult growth hormone deficiency: an Endocrine Society clinical practice guideline. J Clin Endocrinol Metab 2011; 96: 1587-609.

7. Longobardi S, Merola B, Pivonello R, Di Rella F, Di Somma C, Colao A, Ghigo E, Camanni F, Lombardi G. Reevaluation of growth hormone $(\mathrm{GH})$ secretion in 69 adults diagnosed as GH-deficient patients during childhood. J Clin Endocrinol Metab 1996; 81: 1244-7.

8. Cacciari E, Tassoni P, Parisi G, Pirazzoli P, Zucchini S, Mandini M, Cicognani A, Balsamo A. Pitfalls in diagnosing impaired growth hormone $(\mathrm{GH})$ secretion: retesting after replacement therapy of 63 patients defined as GH deficient. J Clin Endocrinol Metab 1992; 74: 1284-9.

9. Wacharasindhu S, Cotterill AM, Camacho-Hübner C, Besser GM, Savage MO. Normal growth hormone secretion in growth hormone insufficient children retested after completion of linear growth. Clin Endocrinol (Oxf) 1996; 45: 5536.

10. Ho KK; 2007 GH Deficiency Consensus Workshop Participants. Consensus guidelines for the diagnosis and treatment of adults with GH deficiency II: a 
statement of the GH Research Society in association with the European Society for Pediatric Endocrinology, Lawson Wilkins Society, European Society of Endocrinology, Japan Endocrine Society, and Endocrine Society of Australia. Eur J Endocrinol 2007; 157: 695-700.

11. Yuen KC, Cook DM, Sahasranam P, Patel P, Ghods DE, Shahinian HK, Friedman TC. Prevalence of GH and other anterior pituitary hormone deficiencies in adults with nonsecreting pituitary microadenomas and normal serum IGF-1 levels. Clin Endocrinol (Oxf) 2008; 69: 292-8.

12. Agha A, Rogers B, Sherlock M, O'Kelly P, Tormey W, Phillips J, Thompson CJ. Anterior pituitary dysfunction in survivors of traumatic brain injury. $\mathrm{J}$ Clin Endocrinol Metab 2004; 89: 4929-36.

13. Tanrıverdi F, Unluhızarcı K, Coksevim B, Selçuklu A, Casanueva FF, Kelestimur F. Kickboxing sport as a new cause of traumatic brain injury-mediated hypopituitarism. Clin Endocrinol (Oxf) 2007; 66: 360-6.

14. Schneider HJ, Schneider M, Kreitschmann-Andermahr I, Tuschy U, Wallaschofski H, Fleck S, Faust M, Renner CI, Kopczak A, Saller B, Buchfelder M, Jordan M, Stalla GK. Structured assessment of hypopituitarism after traumatic brain injury and aneurysmal subarachnoid hemorrhage in 1242 patients: the German interdisciplinary database. J Neurotrauma 2011; 28: 1693-8.

15. Tanriverdi F, Unluhizarc1 K, Kelestimur F. Pituitary function in subjects with mild traumatic brain injury: a review of literature and proposal of a screening strategy. Pituitary 2010; 13: 146-53.

16. Darzy KH, Aimaretti G, Wieringa G, Gattamaneni HR, Ghigo E, Shalet SM. The usefulness of the combined growth hormone $(\mathrm{GH})$-releasing hormone and arginine stimulation test in the diagnosis of radiation-induced GH deficiency is dependent on the post-irradiation time interval. J Clin Endocrinol Metab 2003; 88: 95-102.

17. Miller KK, Wexler T, Fazeli P, Gunnell L, Graham GJ, Beauregard C, Hemphill L, Nachtigall L, Loeffler J, Swearingen B, Biller BM, Klibanski A. Growth hormone deficiency after treatment of acromegaly: a randomized, placebocontrolled study of growth hormone replacement. J Clin Endocrinol Metab 2010; 95: 567-77.

18. Hartman ML, Weltman A, Zagar A, Qualy RL, Hoffman AR, Merriam GR. Growth hormone replacement therapy in adults with growth hormone deficiency improves maximal oxygen consumption independently of dosing regimen or physical activity. J Clin Endocrinol Metab 2008; 93: 125-30.

19. Götherström G, Elbornsson M, Stibrant-Sunnerhagen K, Bengtsson BA, Johannsson G, Svensson J. Muscle strength in elderly adults with GH deficiency after 10 years of GH replacement. Eur J Endocrinol 2010; 163: 207-15.

20. Holmes SJ, Whitehouse RW, Swindell R, Economou G, Adams JE, Shalet SM. Effect of growth hormone replacement on bone mass in adults with adult onset growth hormone deficiency. Clin Endocrinol (Oxf) 1995; 42: 627-33.

21. Shalet SM, Shavrikova E, Cromer M, Child CJ, Keller E, Zapletalová J, Moshang T, Blum WF, Chipman JJ, Quigley CA, Attanasio AF. Effect of growth hormone (GH) treatment on bone in postpubertal GH-deficient patients: a 2-year randomized, controlled, dose-ranging study. J Clin Endocrinol Metab 2003; 88: 4124-9.

22. Baroncelli GI, Bertelloni S, Sodini F, Saggese G. Acquisition of bone mass in normal individuals and in patients with growth hormone deficiency. J Pediatr Endocrinol Metab 2003; 16: 327-35.

23. van der Klaauw AA, Pereira AM, Rabelink TJ, Corssmit EP, Zonneveld AJ, Pijl H, de Boer HC, Smit JW, Romijn JA, de Koning EJ. Recombinant human GH replacement increases CD34+ cells and improves endothelial function in adults with GH deficiency. Eur J Endocrinol 2008; 159: 105-11.

24. Sesmilo G, Biller BM, Llevadot J, Hayden D, Hanson G, Rifai N, Klibanski A. 
Effects of growth hormone administration on inflammatory and other cardiovascular risk markers in men with growth hormone deficiency. A randomized, controlled clinical trial. Ann Intern Med 2000 18; 133: 111-22.

25. Colao A, Di Somma C, Salerno M, Spinelli L, Orio F, Lombardi G. The cardiovascular risk of GH-deficient adolescents. J Clin Endocrinol Metab 2002; 87: 3650-5.

26. Schneider HJ, Klotsche J, Wittchen HU, Stalla GK, Schopohl J, Kann PH, Kreitschmann-Andermahr I, Wallaschofski H; German KIMS board and of the DETECT study. Effects of growth hormone replacement within the KIMS survey on estimated cardiovascular risk and predictors of risk reduction in patients with growth hormone deficiency. Clin Endocrinol (Oxf) 2011; 75: 825-30.

27. Attanasio AF, Lamberts SW, Matranga AM, Birkett MA, Bates PC, Valk NK, Hilsted J, Bengtsson BA, Strasburger CJ. Adult growth hormone (GH)-deficient patients demonstrate heterogeneity between childhood onset and adult onset before and during human GH treatment. Adult Growth Hormone Deficiency Study Group. J Clin Endocrinol Metab 1997; 82: 82-8.

28. Abs R, Mattsson AF, Bengtsson BA, Feldt-Rasmussen U, Góth MI, KoltowskaHäggström M, Monson JP, Verhelst J, Wilton P; KIMS Study Group. Isolated growth hormone $(\mathrm{GH})$ deficiency in adult patients: baseline clinical characteristics and responses to $\mathrm{GH}$ replacement in comparison with hypopituitary patients. A sub-analysis of the KIMS database. Growth Horm IGF Res 2005; 15: 349-59.

29. Falutz J. Growth hormone and HIV infection: contribution to disease manifestations and clinical implications. Best Pract Res Clin Endocrinol Metab 2011; 25: 517-29.

30. Burman P, Johansson AG, Siegbahn A, Vessby B, Karlsson FA. Growth hormone (GH)-deficient men are more responsive to GH replacement therapy than women. J Clin Endocrinol Metab 1997; 82: 550-5.

31. Losa M, Scavini M, Gatti E, Rossini A, Madaschi S, Formenti I, Caumo A, Stidley CA, Lanzi R. Long-term effects of growth hormone replacement therapy on thyroid function in adults with growth hormone deficiency. Thyroid 2008; 18 : 1249-54.

32. Giavoli C, Libé R, Corbetta S, Ferrante E, Lania A, Arosio M, Spada A, BeckPeccoz P. Effect of recombinant human growth hormone $(\mathrm{GH})$ replacement on the hypothalamic-pituitary-adrenal axis in adult GH-deficient patients. J Clin Endocrinol Metab 2004; 89: 5397-401.

33. Holmes SJ, Shalet SM. Which adults develop side-effects of growth hormone replacement? Clin Endocrinol (Oxf) 1995; 43: 143-9.

34. Hansen R, Koller EA, Malozowski S. Full remission of growth hormone (GH)induced retinopathy after GH treatment discontinuation: long-term follow-up. J Clin Endocrinol Metab 2000; 85: 2627.

35. Malozowski S, Tanner LA, Wysowski DK, Fleming GA, Stadel BV. Benign intracranial hypertension in children with growth hormone deficiency treated with growth hormone. J Pediatr 1995; 126: 996-9.

36. Cohn L, Feller AG, Draper MW, Rudman IW, Rudman D. Carpal tunnel syndrome and gynaecomastia during growth hormone treatment of elderly men with low circulating IGF-I concentrations. Clin Endocrinol (Oxf) 1993; 39: 41725. 